\title{
Modelo de gestión para un control de calidad eficiente en edificios de nueva planta
}

\section{Management Model for efficient quality control in new buildings}

\author{
C. E. Rodríguez-Jiménez ${ }^{(*)}$, J. M. Macías-Bernal ${ }^{(*)}$, R. Lucas-Ruiz ${ }^{(*)}$
}

\section{RESUMEN}

La gestión del control de calidad en cada proceso edificatorio se plantea habitualmente en España desde distintos niveles de exigencia.

El presente trabajo tiene por objeto obtener un modelo de referencia para comparar el control del proceso edificatorio realizado sobre un producto concreto (edificio) y poder evaluar su nivel de garantía.

Para ello recurrimos a fuentes especializadas así como al estudio de 153 casos reales de expedientes de control de calidad utilizando una metodología del tipo multicriterio. Se aplican técnicas conducentes a una valoración concreta (objetiva) de los parámetros de entrada mediante el método Delphi (consulta a 17 expertos), cuyo tratamiento matricial con la herramienta Fuzzy-QFD condensa referencias numéricas a través de un reparto de pesos entre las funciones seleccionadas y sus correspondientes factores intervinientes.

El modelo así obtenido (M153) es útil como referente óptimo de las actuaciones de control que conllevan el cumplimiento de las expectativas de calidad.

Palabras clave: control de calidad; calidad en la edificación; gestión; Fuzzy-QFD.

\section{ABSTRACT}

The management of the quality control of each building process is usually set up in Spain from different levels of demand. This work tries to obtain a model of reference, to compare the quality control of the building process of a specific product (building), and to be able to evaluate its warranty level.

In the quest of this purpose, we take credit of specialized sources and 153 real cases of Quality Control were carefully revised using a multi-judgment method. Applying different techniques to get a specific valuation (impartial) of the input parameters through Delphi's method (17 experts query), whose matrix treatment with the Fuzzy-QFD tool condenses numerical references through a weighted distribution of the selected functions and their corresponding conditioning factors.

The model thus obtained (M153) is useful in order to have a quality control reference to meet the expectations of the quality.

Keywords: quality control; building quality; management; Fuzzy-QFD.

(*) Departamento de Construcciones Arquitectónicas II. Universidad de Sevilla, Sevilla (España).

Persona de contacto/Corresponding author: ceugenio@us.es (C.E. Rodríguez-Jiménez)

ORCID: http://orcid.org/oooo-0003-3792-3389 (C. E. Rodríguez-Jiménez); http://orcid.org/oooo-0001-6073-9745

(J. M. Macías-Bernal); http://orcid.org/oooo-0003-4771-6068 (R. Lucas-Ruiz)

Cómo citar este artículo/Citation: Rodríguez-Jiménez,C. E., Macías-Bernal, J. M., Lucas-Ruiz, R. (2017). Modelo de gestión para un control de calidad eficiente en edificios de nueva planta. Informes de la Construcción, 69(547): e204, doi: http://dx.doi.org/10.3989/ic.16.036.

Copyright: (C) 2017 CSIC. Licencia / License: Salvo indicación contraria, todos los contenidos de la edición electrónica de Informes de la Construcción se distribuyen bajo una licencia de uso y distribución Creative Commons Attribution License (CC BY) Spain 3.o. 


\section{INTRODUCCIÓN}

El control de calidad en edificios de nueva planta, entendido como el conjunto de las actuaciones que llevan a cabo las organizaciones de control, posee en España un marco regulatorio y funcional que deja muy abierto el diseño de su aplicación.

En consecuencia existe una considerable dispersión en la tipología y alcance de las actuaciones, fruto de aplicar diferentes criterios en cada caso. Además este planteamiento flexible propicia que determinadas circunstancias exógenas a los requisitos técnicos, tales como los condicionantes contractuales, acaben marcando gran parte del trazado a seguir.

De este modo, en muchos procesos edificatorios las posibilidades del control acaban reduciéndose a la subcontratación de entidades específicas para realizar únicamente algunos ensayos físicos sobre muestras de materiales, aspecto que han detallado autores como Garrido (1). Sin embargo, las voces históricas en la materia, como Ishikawa, ya dejaban claro en su doctrina que «el fin del control de calidad es garantizar la calidad por medio de su control» (2), es decir, nos encontramos con una realidad en la que la mayor parte de los planes de control desarrollados se distancian de su objetivo, pues no agotan todo el potencial del control para reducir fallos y lograr un producto de calidad.

El presente trabajo recoge los resultados de la investigación llevada a cabo con el objetivo de mejorar ese panorama. Se pretende proporcionar a los responsables de gestión de las promociones arquitectónicas un patrón para las organizaciones de control implicadas, el cual debe posibilitar la culminación de su cometido en pro de la calidad. De tal modo, partiendo de los condicionantes existentes y considerando los recursos disponibles, la metodología utilizada permite recomponer los diversos planteamientos que se han venido utilizando hasta la fecha, conformando así un único modelo eficiente que sirva de referencia para el diseño de las actividades de control.

\section{ESTADO DE LA CUESTIÓN}

\subsection{Marco general de participantes}

La Ley 38/1999 de Ordenación de la Edificación (en adelante LOE) es, sin duda, el elemento esencial que enmarca toda la regulación existente. De cara a asegurar la calidad el texto legislativo establece el marco de funciones y responsabilidades en cuanto al control, designando los agentes que deben dedicarse exclusivamente al control del proceso:

- Los laboratorios de control de calidad, para realizar ensayos y pruebas de materiales y sistemas.

- Las entidades de control de calidad (ECC), cuya misión es el control de la calidad del proyecto, la ejecución y los materiales.

La ley otorga a estos controladores un papel de asistentes técnicos y, por tanto, no decisorios, centrando las responsabilidades finales en cuanto al control en el Proyectista y el Director de Ejecución en sus ámbitos respectivos.

Un tercer agente con misión exclusivamente controladora lo constituyen los Organismos de Control Técnico (OCT), no contemplados directamente en la LOE, pero surgidos como una derivada de la misma a tenor de la evaluación de riesgos para baremar las condiciones de la póliza del Seguro de Daños (3) que debe cubrir la garantía de estabilidad en los edificios residenciales, pues hasta la fecha y según el artículo 19 de la citada ley es prescriptivo asegurar únicamente los daños de origen geotécnico y estructural.

\subsection{Requisitos legislativos para el control}

En el plano de ordenación del sector la LOE sólo fija las tres líneas básicas (seguridad, habitabilidad y funcionalidad) que el resto de normas están llamadas a desarrollar. Sin embargo, el tratamiento dado al control de calidad por la legislación prescriptiva española suscita algunas dudas al no incluir procedimientos precisos de control en todas las facetas del proceso edificatorio.

Si atendemos a la normativa técnica principal debemos considerar en primer lugar el Código Técnico de la Edificación (CTE). Éste posee un carácter prestacional, que Serra (4) ubica en el nivel mínimo de requisitos que puede fijar una norma (objetivos o intereses esenciales), concediendo libertad a los agentes responsables del proceso para establecer las estrategias de control que conduzcan al nivel de prestaciones requerido. El mejor ejemplo de ello es, quizá, la ausencia de pruebas obligatorias para estanqueidad de cubiertas (que ya figuraban en las antiguas normas NBE), dejando en manos de la Dirección Facultativa la forma de verificar el grado de impermeabilidad prescrito.

La instrucción EHE, en cambio, sí posee un completo alcance que incluye las directrices a cumplir, el desarrollo del control, los criterios de aceptación y las decisiones derivadas. Pero obviamente su ámbito de aplicación se circunscribe exclusivamente a los ensayos y control de ejecución de estructuras de hormigón armado y pretensado.

También cabe reseñar la reglamentación para la Calidad y la Seguridad Industrial (RD 2200/95 y disposiciones afines) que establece el marco de funcionamiento de los procesos de certificación y la inspección reglamentaria, los cuales se configuran como un medio adecuado para que el productor pueda trasladar la garantía necesaria al utilizador.

Por último, aunque se trata de una norma no prescriptiva, su amplia consolidación en el sector de la construcción hace digna la mención a la norma UNE-EN ISO 9001 para requisitos de Sistemas de Gestión de la Calidad. Si bien hay que tener presente que se trata de un texto enfocado para su implantación en el seno de una empresa u organización, por lo que sus requerimientos no son directamente aplicables al complejo sistema de actores y etapas que conforman el proceso edificatorio completo.

\section{METODOLOGÍA}

La investigación se ha centrado en la configuración de un modelo que incorpore, por un lado, los principios fundamentales para el desarrollo útil del control de calidad, y por otro, el conjunto de actuaciones necesarias para mantener bajo control la totalidad del proceso técnico de construcción de edificios.

Para ello se siguen los siguientes pasos: 
A. Fase teórica. Elección de las herramientas teóricas a emplear.

B. Fase experimental. Que se compone a su vez de tres etapas:

- Selección de factores condicionantes del control.

- Determinación de funciones operativas.

- Ponderación de factores y funciones.

C. Propuesta del modelo. Donde se establecen las bases para la propuesta de referencia y su viabilidad tras integrar resultados empíricos y técnicas instrumentales.

\section{FUNDAMENTOS TEÓRICOS}

Para la elección de los fundamentos metodológicos teóricos se han tenido en cuenta que las herramientas a emplear deben satisfacer dos requisitos esenciales:

- Contar con una trayectoria contrastada en cuanto a tratamiento de la calidad y el control.

- Estar centradas en una gestión de productos que priorice la optimización de recursos y la minimización de fallos.

A partir de estos dos supuestos las directrices expuestas en la normativa de aplicación y en otras fuentes específicas nos llevan a considerar una metodología multicriterio, la cual se apoya en los tres sistemas que exponemos a continuación.

\subsection{La técnica QFD}

La técnica QFD (Quality Function Deployment o Despliegue de la Función Calidad) está incluida dentro de la metodología de gestión Seis Sigma descrita en la norma UNE-ISO 13053-2:2012. Se trata de un proceso analítico para determinar cómo las características de un producto o servicio (a las cuales denomina genéricamente los «Cómo») cumple las expectativas que debe salvar (denominadas los «Qué»).

Se lleva a cabo a través de una sencilla matriz donde se incorporan las valoraciones numéricas de cada una de las entradas.

La adaptación a nuestro caso de este método nos lleva a plantear como entradas de la matriz los factores y funciones del control a seleccionar (Figura 1).

\section{Funciones (¿Cómo?)}

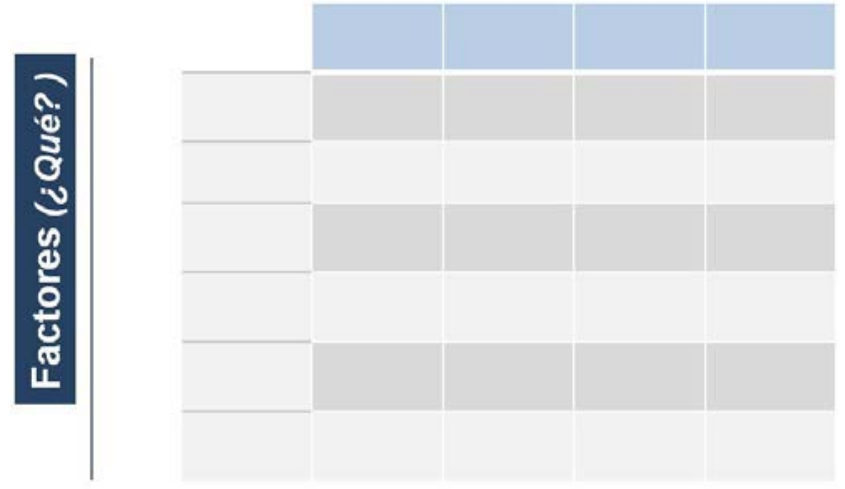

Figura 1. Esquema matriz QFD con las entradas del modelo.
La técnica QFD conduce a ponderar cada una de estas entradas, para lo cual nos apoyamos en los métodos Delphi y el análisis Fuzzy tal como exponen, entre otros, las investigaciones de Osorio et al. (5).

\subsection{El método Delphi}

El contraste de los datos e información obtenidos en un proceso puede someterse al llamado Grupo de Consenso, o Método Delphi, incluido en la norma UNE-ISO 31010:2011 sobre técnicas de apreciación del riesgo, mediante el cual se somete el problema a la opinión de un grupo de especialistas externos que colaboran para converger hacia una valoración fundamentada.

Las consultas se realizan de forma independiente entre los participantes, que sólo contactan con el coordinador y nunca entre ellos, logrando así la una objetividad máxima.

\subsection{Análisis Fuzzy}

La lógica difusa (Fuzzy Logic), introducida por L. Zadeh en 1965 (6), supone un paso que va más allá de la lógica tradicional. En la actualidad los análisis matemáticos con lógica difusa se establecen como un avanzado método de interpretación y tratamiento semántico derivado del pensamiento humano.

Así la pertenencia de una realidad dada a una de las llamadas etiquetas lingüísticas (como, por ejemplo, frío/calor, alto/ bajo, bueno/malo, etc.) no es siempre unívoca, sino que puede ser variable en función de la percepción personal de quien emite el juicio.

De tal modo unas determinadas sensaciones térmicas son clasificadas en la lógica tradicional simplemente como temperaturas altas o bajas, pero en la lógica borrosa contempla una zona difusa donde unos hablaran de alta y otros de baja, por tanto la pertenencia a estos conceptos (llamados conjuntos difusos o fuzzy set) puede ser variable. Así la lógica difusa establece que una determinada temperatura intermedia tiene un cierto grado de pertenencia a la etiqueta «temperatura alta» al mismo tiempo que a la de «temperatura baja», según las diferentes interpretaciones.

A partir de aquí el procedimiento debe contar con unas reglas de inferencia, del tipo «si la temperatura es baja, se encenderá la calefacción", las cuales servirán para obtener unas salidas difusas a las entradas planteadas. Estos parámetros de salida, tras un mecanismo desdifusor, se presentan como resultados finales (siguiendo el ejemplo sería el valor de temperatura a la que debemos fijar el termostato).

\section{FUNDAMENTOS EMPÍRICOS}

\subsection{Selección de factores determinantes del control}

La recopilación de factores determinantes para el control se ha llevado a cabo a partir de una selección de fuentes españolas con amplia trayectoria en esta disciplina. El resultado es un conjunto bibliográfico publicado por un elenco de autores nacionales de indiscutible impacto en la materia. Sin duda, el conocimiento y la experiencia transmitida a través de su obra han permitido establecer gran parte de las líneas maestras para la calidad en construcción y su control durante las últimas décadas. Nos referimos a: 
- Álvaro García Meseguer.

- José Calavera Ruiz.

- Antonio Garrido Hernández.

- Faustino Merchán Gabaldón.

- La Asociación Española para la Calidad.

El mapa de aspectos condicionantes para el control que se desprende de dichos autores, así como los otros parámetros contemplados en la normativa de referencia, sirve para establecer el compendio de los 7 factores condicionantes de entrada al modelo que se detallan seguidamente.

\section{1. ${ }^{\text {er }}$ factor: muestreo}

Es una actividad de planificación que marca la extensión, distribución y representatividad de las muestras objeto de control en cada faceta.

La estadística proporciona herramientas adaptadas que son de aplicación habitual en producción industrial en serie, por lo que suele ser la tónica en la fabricación de materiales y equipos. Pero su aplicación en los procesos de proyecto y ejecución de obra choca con numerosos condicionantes (económicos, organizativos, temporales, etc.) que impiden realizar un muestreo de amplitud suficiente. Es fácil que los planes de control seguidos en edificación contemplen, salvo en las unidades estructurales, controles de materiales o pruebas de funcionamiento con un número de inspecciones o ensayos muy reducido (1 o 2 a lo sumo en muchas unidades).

\section{2. ${ }^{\circ}$ factor: coordinación entre etapas}

Es el factor que marca la trazabilidad del control durante todo el proceso.

Se refiere a la necesidad de interrelacionar los resultados del control en cada uno de los procesos y fases, para lograr un abanico de resultados configurado por la suma de los distintos implicados. García-Meseguer (7) resume perfectamente el concepto al describir las relaciones entre el control de producción y de recepción en «los modos de control».

Si se realiza de forma coordinada entre los agentes participantes, el volumen y naturaleza de las características del producto puede llegar a ser muy completa al poder aportarse determinaciones complementarias realizadas por productores, certificadores, etc., optimizando las actuaciones de control. Por ejemplo, la determinación del comportamiento al fuego de los elementos constructivos es un procedimiento demasiado costoso para incluirlo en el presupuesto de control durante la construcción del edificio, por lo que parece razonable que el fabricante asuma la realización de estas pruebas.

\section{$3{ }^{\text {er }}$ factor: criterios de aceptación y rechazo}

La necesidad de contar con fundamentos fiables que avalen las decisiones derivadas del control es determinante para culminar el proceso con acierto. Los parámetros límite a contemplar para las características de los materiales, las condiciones de ejecución, de ensayo o la interpretación de resultados, entre otros, son aspectos que deberían figurar definidos en cada caso. Como se ha citado al tratar el estado del arte, la normativa actual de obligado cumplimiento no aporta siempre los requisitos funcionales y operativos necesarios.
$4 \cdot{ }^{\circ}$ y $5 \cdot{ }^{\circ}$ factores: retroalimentación y vulnerabilidad

Ambos factores coinciden en cuanto a su vinculación con el periodo de vida útil y la durabilidad del edificio. Nos encontramos ante dos rasgos que apoyan la prevención:

- Por un lado el factor retroalimentación se refiere al estudio de las reclamaciones y fallos que se han suscitado durante la vida útil de los edificios para ser considerada como elementos de mejora en los sucesivos procesos constructivos y por ende en su control.

- De otra parte el factor vulnerabilidad, que es incorporado a la selección por el equipo de investigación como derivada de la anterior y en línea con investigaciones anteriores (8) (9). Es obvio que las anomalías de los elementos del edificio durante su funcionamiento están relacionadas con su mayor o menor vulnerabilidad, es decir, es necesario tener presente el riesgo de alteración que tiene cada unidad para diseñar una estrategia de control adaptada. La vulnerabilidad está condicionada tanto por elementos integrados en el propio edificio (una mayor exposición, por ejemplo) como por aspectos del entorno (por ejemplo, la pluviometría).

\section{6. ${ }^{\circ}$ factor: independencia}

En edificación es tradicional de que el control sea asumido sólo por el propio productor (proyectista, fabricante o constructor). Es obvio que quien desempeña una actividad tiene que mantenerla bajo control, pero no es menos lógico considerar que la admisibilidad de un elemento debe recaer en última instancia sobre alguien ajeno a los intereses del producto.

No es un asunto muy recurrente en la bibliografía, pero existen menciones muy relevantes como la reflejada en los comentarios al artículo 78 de la instrucción EHE-o8 recomendando que el control se contrate y abone por la propiedad.

También Garrido Hernández hace hincapié en el tema y justifica la desvinculación del control al agente controlado: «La razón es clara: que el agente controlado no pueda contar con ninguna posibilidad de influir indebidamente sobre el agente controlador» (10).

En consecuencia es razonable aplicar al control una estructura en que el ejecutor aporte «la carga de la prueba» (11) con un completo autocontrol que sea contrastado sistemáticamente por un controlador externo independiente que complementa al anterior. Esta estrategia, muy habitual en obras civiles promovidas por la administración, proporciona un marco que garantiza la objetividad del proceso controlador.

\section{7. ${ }^{\circ}$ factor: motivación}

La actividad de la construcción posee una gran dependencia de la mano de obra, no pudiendo escapar a esta circunstancia el control de calidad. Esta influencia de las personas convierte a la motivación en un factor determinante en los resultados. La motivación debe entenderse aquí como un término que condensa un conjunto de conceptos vinculados a la actitud y aptitud de las personas en relación a su trabajo en el control.

La Dra. Navarro ha realizado investigaciones en este campo para los técnicos del sector de la construcción (12), cuyas conclusiones ponen de manifiesto que la mayor parte de los 
elementos motivadores son intrínsecos al trabajo (características de las tareas o la autorrealización) mientras los elementos negativos siempre provienen de fuentes externas al propio trabajo (condiciones del trabajo o estabilidad).

\subsection{Determinación de funciones operativas del control}

Para determinar las funciones operativas realizamos un amplio estudio de la documentación generada por las organizaciones de control de calidad en la construcción de 153 edificios en la provincia de Sevilla durante el periodo comprendido entre 2007 y 2013. Se trata en todos los casos de promociones de nueva planta que han sido terminadas en ese intervalo temporal y donde encontramos todo tipo de usos, residencial, docente, administrativo, sanitario, religioso, etc.

La primera categorización que surge del análisis de los expedientes atiende a la extensión cualitativa del control llevado a cabo desde el punto de vista de la amplitud y modalidad del mismo. Así se establecen tres categorías con la siguiente cuantificación:

- Control básico (75 expedientes; 49,0 \% del total).

- Control ampliado (44 expedientes; 28,8 \% del total).

- Control total (34 expedientes; 22,2 \% del total).

A partir de aquí el procedimiento de trabajo con los expedientes pasa por la elección de unos representantes de cada una de las tres categorías donde se estudian pormenorizadamen- te todas las intervenciones realizadas, sus resultados, consecuencias y otras derivadas (Tabla 1).

Para el tratamiento del extenso conjunto de datos obtenidos de estudio general de la totalidad de expedientes y del examen pormenorizado a los representantes destacados se utiliza la técnica de análisis estratégico DAFO. Las principales ideas que surgen de la información recabada se reflejan en el cuadro resumen DAFO (Tabla 2).

El estudio y análisis descrito para los 153 expedientes reales de control de calidad en edificios de nueva planta y la consulta de diversas fuentes especializadas conduce a la propuesta de 7 funciones operativas o campos de actuación útiles para el modelo cuya caracterización se resume seguidamente:

\section{1. ${ }^{a}$ función: control del encargo}

Hace referencia a la verificación de las condiciones técnicas en la contratación así como a la organización de los diferentes agentes y procesos participantes.

Está presente de forma muy minoritaria en los expedientes estudiados, pero su importancia específica y la trascendencia en el resto de funciones motivan su inclusión como factor de gran calado para el control.

García-Meseguer alude a este punto con un axioma retórico que lo resume: «sólo el que sabe lo que quiere sabe pedir lo que quiere» (13).

Tabla 1. Muestra representativa de los 153 expedientes de control estudiados.

\begin{tabular}{|c|c|c|}
\hline n.o & Promoción & n. ${ }^{\circ}$ en el estudio \\
\hline 1 & 108 viviendas Polígono Aeropuerto, Sevilla & Expediente $n .^{\circ} 92$. \\
\hline 2 & Edificio religioso, Distrito Sur, Sevilla. & Expediente n. ${ }^{\circ} 100$. \\
\hline 3 & 1 vivienda unifamiliar, Villaverde del Río, Sevilla & Expediente n. ${ }^{\circ} 102$. \\
\hline 4 & 21 viviendas, Coria del Río, Sevilla & Expediente n. ${ }^{\circ} 104$. \\
\hline 5 & 78 viviendas, Camas, Sevilla & Expediente $n .^{\circ} 59$. \\
\hline 6 & 11 viviendas sector Su Eminencia, Sevilla & Expediente $n .^{\circ} 67$. \\
\hline 7 & Centro Educativo, Brenes, Sevilla & Expediente n. ${ }^{\circ} 93$. \\
\hline 8 & 1 vivienda, Árbol Gordo, Sevilla & Expediente n. ${ }^{\circ} 136$. \\
\hline 9 & 46 viviendas en Barriada La Corza, Sevilla & Expediente n. ${ }^{\circ} 8$. \\
\hline 10 & Centro Educativo, Santiponce, Sevilla & Expediente n. ${ }^{\circ} 49$. \\
\hline 11 & 37 viviendas, Bami-Su Eminencia, Sevilla & Expediente n. ${ }^{\circ} 90$. \\
\hline 12 & 52 viviendas, Torreblanca, Sevilla & Expediente.$^{\circ}{ }^{0} 118$. \\
\hline
\end{tabular}

Tabla 2. Resumen ideas DAFO tras análisis de los 153 expedientes.

\begin{tabular}{|c|c|c|}
\hline & - & + \\
\hline$\frac{\overbrace{0}^{n}}{2}$ & $\begin{array}{l}\text { DEBILIDADES: } \\
\text { 1. Distribución del control en el proceso } \\
\text { 2. Resultados sin pronunciamiento } \\
\text { 3. Dimensionado sin criterios técnicos } \\
\text { 4. Falta de orientación al usuario }\end{array}$ & $\begin{array}{l}\text { FORTALEZAS: } \\
\text { 9. Asistencias técnicas eficaces } \\
\text { 10. Porcentaje casos con control total } \\
\text { 11. Control materiales EHE ejemplar } \\
\text { 12. Cumplimiento del plan de control } \\
\text { 13. Organización documental }\end{array}$ \\
\hline $\begin{array}{l}\frac{n}{0} \\
\frac{0}{0} \\
0 \\
2\end{array}$ & $\begin{array}{l}\text { AMENAZAS: } \\
\text { 5. Control como mera partida de obra } \\
\text { 6. Desviación del control al asegurador } \\
\text { 7. Falta de atención a los certificados } \\
\text { 8. Saturación comunicativa }\end{array}$ & $\begin{array}{l}\text { OPORTUNIDADES: } \\
\text { 14. Incremento tecnológico } \\
\text { 15. Racionalizar el control de materiales } \\
\text { 16. Certificación de edificios completos }\end{array}$ \\
\hline
\end{tabular}


Calavera justifica esta actividad por la necesidad de incorporar a la cadena del control «un eslabón más en cada extremo» (14) en relación a sumar un control del encargo al inicio y un control de la entrega al final del proceso (este último se abordará más tarde).

Podría llamarse también control organizativo, aunque la bibliografía suele denominarlo tal como se recoge en la presente investigación. Tiene una estrecha unión con el promotor, al ser el agente que realiza los contratos principales y es responsable de la programación general.

\section{2. ${ }^{\mathrm{a}}$ función: control de proyecto}

La supervisión técnica del proyecto de ejecución es una actividad extendida en nuestro entorno (aparece en el 80,4 \% de los expedientes analizados), si bien sólo en 36 de las 153 promociones estudiadas este control es voluntario, es decir, se realiza fuera de los requisitos impuestos a tenor del seguro decenal de daños derivado de las garantías de estabilidad de la LOE.

Verificar el proyecto técnico del edificio es indiscutiblemente una importante actividad a considerar en el control de un edificio. Muchos estudios estadísticos de patología en edificación reflejan que sobre el proyecto recaen la mayor parte de las causas de las mismas (15) (16).

\section{$3 \cdot{ }^{\mathrm{a}}$ función: control de ejecución}

$\mathrm{Al}$ igual que en el caso del control del proyecto, el número de expedientes del análisis donde las organizaciones de control realizan este control de forma no vinculada al seguro decenal es escaso (sólo 42 de los 153 casos).

El control de la ejecución es una misión ligada a la dirección facultativa de la obra, cuyos miembros ejercen de máximos decisores en materia de control, siendo la actividad de los organismos de control un apoyo cuantitativo en el grueso entramado de operaciones y diversidad tecnológica que supone la ejecución material de un edificio. Además una completa supervisión de la ejecución se justifica también por tratarse de una fase con gran dependencia de la mano de obra, la cual realiza proyectos no repetitivos con el consiguiente aumento de probabilidad de errores.

\section{$4 \cdot{ }^{\mathrm{a}}$ función: control de materiales}

Es la función del control con mayor arraigo en edificación. Principalmente los materiales componentes de las estructuras de hormigón poseen una tradición derivada de las prescripciones incluidas en las sucesivas Instrucciones para estructuras de hormigón armado, desde décadas atrás. Así se pone de manifiesto en los 153 expedientes que nos sirven de base, en los que es constante este tipo de control.

Esta universalización del control de materiales tiene también su reflejo en la disminución de su responsabilidad en las causas de patología, lo que avala la eficacia del control para alcanzar la calidad.

\section{$5 \cdot^{\mathrm{a}}$ función: pruebas de funcionamiento}

Suponen el verdadero banco de pruebas del edificio, pues miden prestaciones reales de las unidades probadas tal como han sido construidas.
En general están centradas en las instalaciones y la estanqueidad de la envolvente, pero existen técnicas para verificar gran parte de los aspectos que integran una edificación, tales como acústica, transmitancia térmica, eficiencia energética, pruebas de carga, renovaciones de aire, intensidad de la iluminación, etc.

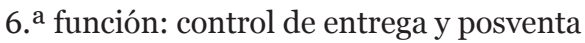

La supervisión de terminaciones del inmueble y la verificación de las incidencias planteadas durante el primer periodo de uso del edificio complementa al apartado de pruebas y supone una conexión del proceso de materialización del edificio con el mantenimiento.

El control de entrega y posventa definido aporta, como aspecto también positivo, una marcada orientación directa al usuario, diferenciándose así del segundo plano en que lo sitúan muchas de las etapas anteriores, según se desprende del análisis DAFO realizado.

\section{7. ${ }^{\text {a }}$ función: asistencia técnica}

Se trata de una labor de gran repercusión para el control, pues permite que el controlador aporte, además de resultados, su punto de vista en los diversos aspectos técnicos para la materialización del edificio.

En concreto podemos señalar seis rasgos que esta actividad suma a la labor de control de calidad:

- Presencia del controlador durante todo el proceso.

- Realización de control documental de certificados, marcados, etc.

- Cauce de voz para el controlador.

- Seguimiento y cumplimiento de los planes de control.

- Aval de uso en nuevas tecnologías.

Se conforma así la participación del agente controlador con su máximo potencial, sirviendo de apoyo eficaz para el resto de los participantes en la totalidad de etapas.

\subsection{Ponderación de entradas al modelo mediante consulta a expertos}

Las entradas diseñadas para el modelo (factores condicionantes y funciones operativas) deben ser cuantificadas en cuanto a sus respectivos pesos de influencia. Utilizamos para ello la consulta a 17 expertos del sector con diferentes perfiles, los cuales nos aportan distintas ópticas del proceso edificatorio.

Partiendo de la matriz QFD de inicio con los dos grupos de entradas, tal como se ha definido en la Figura 1, la consulta se desarrolla en dos etapas:

\section{1. ${ }^{\mathrm{a}}$ consulta a expertos: ponderación de factores}

Los factores determinantes del control dibujan el perfil principal del modelo buscado. Por tanto han sido ponderados de forma prioritaria, con una serie de cuestiones a los expertos acerca de la influencia de cada uno de ellos en la eficiencia del control.

En concreto se propone una escala tipo likert con cinco opciones escalonadas: 
- Muy bajo (MB).

- Bajo (B).

- Medio (M).

- Alto (A).

- Muy alto (MA).

2. ${ }^{\mathrm{a}}$ consulta a expertos: ponderación de factores

La segunda ronda de consulta tiene por objeto determinar el peso de la relación entre cada factor y las diferentes funciones operativas, es decir, el grado en que cada una de estas funciones o actividades pueden colaborar a salvar los factores.

Se forma así un conjunto de 49 interrelaciones a valorar compuestas por parejas de un factor y una función. Se utiliza para ello la misma escala likert de la primera vuelta en la que se ha añadido una valoración nula (S), en línea con el uso habitual de la técnica QFD, dada la variedad de supuestos a ponderar.

La cuantificación del espectro de respuestas dadas a los cuestionarios se lleva a cabo mediante conjuntos difusos. Comenzamos por establecer las funciones difusas más usuales para las etiquetas lingüísticas de la escala likert utilizada (17) (18), dentro de un universo de discurso establecido desde o hasta 10, y fijando en consecuencia funciones de pertenencia triangulares adaptadas al estudio, tal como refleja la Figura 2.

Para los cálculos difusos se emplea el programa XFuzzy 3.0 (19), introduciendo las funciones de pertenencia asociadas a las variables lingüísticas definidas así como las diferentes reglas de inferencia formadas con las 17 respuestas de los respectivos expertos para cada salida buscada.
Como operador «desfuzzification» para pasar de magnitudes borrosas a concretas utilizamos la función «centro del área».

Los resultados concretos obtenidos para las dos fases de la consulta queda como indica la Tabla 3.

Obsérvese como existe un rango de valores muy amplio, obedeciendo a las diferentes situaciones ofrecidas por el binomio factor/función. Por ejemplo, la valoración de 9,8 que ofrece el factor «criterios de aceptación/rechazo» para la función «control de materiales» responde a la gran utilidad de poseer un baremo para la interpretación de resultados de ensayo. En cambio el factor muestreo no participa de forma significativa en el control de encargos a participantes, de ahí su escasa ponderación.

\section{PROPUESTA Y VIABILIDAD DEL MODELO M153}

La propuesta del modelo (al que se ha llamado M153 en referencia al número de expedientes de control utilizados en el estudio) se ha concebido básicamente a través del conjunto de funciones y actividades operativas a desempeñar por las organizaciones de control en edificios de nueva planta, otorgando a cada una de ellas su peso final determinado y recogiendo también la distribución de estas cuantías entre los factores condicionantes derivados de las bases del modelo.

La técnica QFD permite obtener estos pesos a partir de las valoraciones obtenidas con el método Delphi, conformando la matriz final del modelo M153 (Tabla 4).

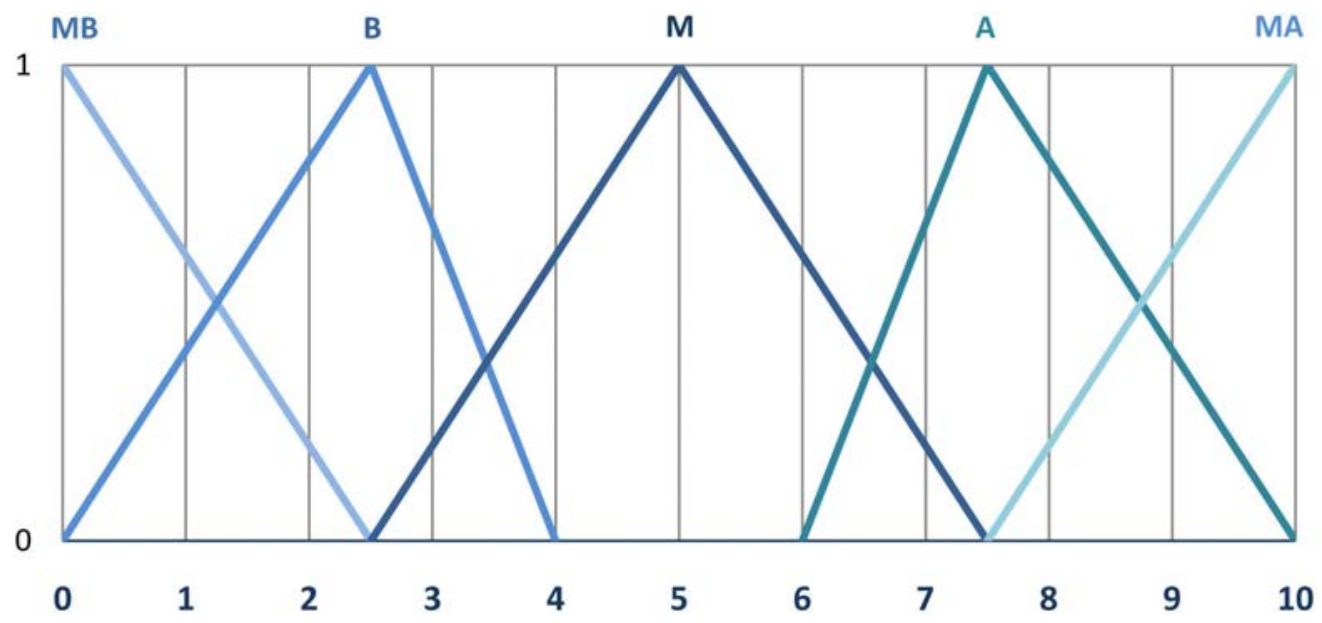

Figura 2. Gráfica de las funciones de pertenencia difusas triangulares asociadas a las etiquetas lingüísticas.

Tabla 3. Resultados finales concretos tras la valoración de factores y funciones.

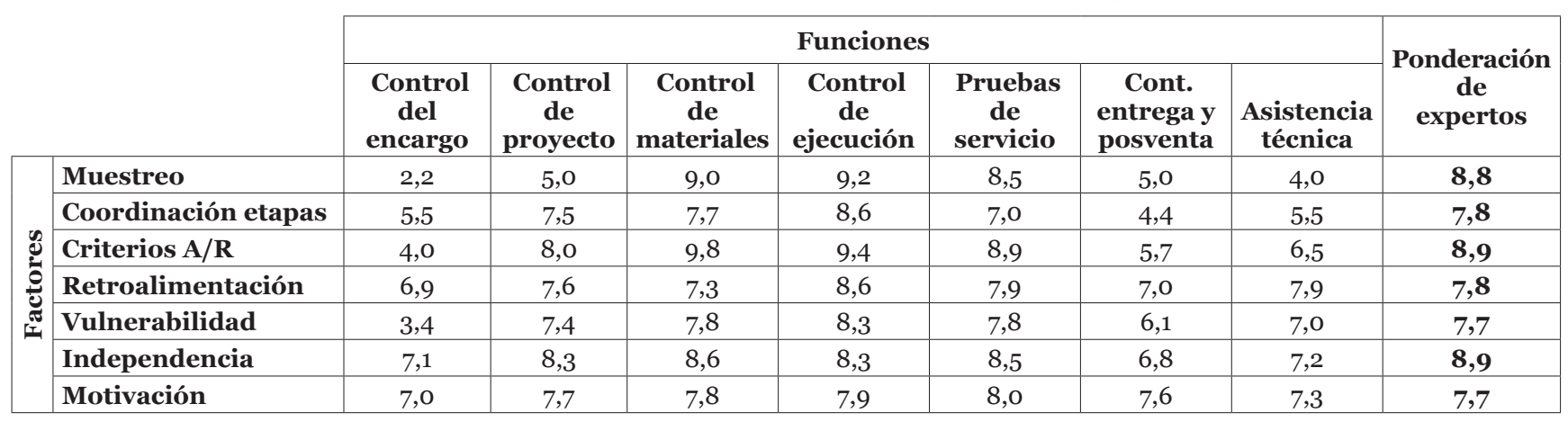


Tabla 4. Matiz final del modelo M153.

\begin{tabular}{|c|c|c|c|c|c|c|c|c|c|c|c|}
\hline & \multicolumn{7}{|c|}{ Funciones } & \multirow[b]{2}{*}{$\begin{array}{l}\text { Ponde- } \\
\text { ración }\end{array}$} & \multirow[b]{2}{*}{$\begin{array}{c}\text { Pond. } \\
\text { Relativa } \\
\%\end{array}$} & \multirow[b]{2}{*}{$\begin{array}{l}\text { Orden } \\
\text { impor- } \\
\text { tancia }\end{array}$} \\
\hline & & $\begin{array}{c}\text { Control } \\
\text { del } \\
\text { encargo }\end{array}$ & $\begin{array}{c}\text { Control } \\
\text { de } \\
\text { proyecto }\end{array}$ & $\begin{array}{c}\text { Control } \\
\text { de mate- } \\
\text { riales }\end{array}$ & $\begin{array}{l}\text { Control } \\
\text { de ejecu- } \\
\text { ción }\end{array}$ & $\begin{array}{c}\text { Pruebas } \\
\text { de } \\
\text { servicio }\end{array}$ & $\begin{array}{c}\text { C. } \\
\text { entrega } \\
\text { y pos- } \\
\text { venta }\end{array}$ & $\begin{array}{c}\text { Asis- } \\
\text { tencia } \\
\text { Técnica }\end{array}$ & & & \\
\hline \multirow{10}{*}{ 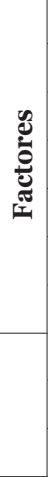 } & Muestreo & 2,2 & 5,0 & 9,0 & 9,2 & 8,5 & 5,0 & 4,0 & 8,8 & 15 & 2 \\
\hline & Coordinación etapas & 5,5 & 7,5 & 7,7 & 8,6 & 7,0 & 4,4 & 5,5 & 7,8 & 14 & 3 \\
\hline & Criterios A/R & 4,0 & 8,0 & 9,8 & 9,4 & 8,9 & 5,7 & 6,5 & 8,9 & 16 & 1 \\
\hline & Retroalimentación & 6,9 & 7,6 & 7,3 & 8,6 & 7,9 & 7,0 & 7,9 & 7,8 & 14 & 3 \\
\hline & Vulnerabilidad & 3,4 & 7,4 & 7,8 & 8,3 & 7,8 & 6,1 & 7,0 & 7,7 & 13 & 4 \\
\hline & Independencia & 7,1 & 8,3 & 8,6 & 8,3 & 8,5 & 6,8 & 7,2 & 8,9 & 16 & 1 \\
\hline & Motivación & 7,0 & 7,7 & 7,8 & 7,9 & 8,0 & 7,6 & 7,3 & 7,7 & 13 & 4 \\
\hline & Evaluación final (absol.) & 295,0 & 423,1 & 480,1 & 497,4 & 467,5 & 349,7 & 371,8 & & & \\
\hline & Evaluación final (\%) & 10 & 15 & 17 & 17 & 16 & 12 & 13 & & & \\
\hline & Orden de importancia & $7^{0}$ & $4^{0}$ & $2^{0}$ & $1^{0}$ & $3^{0}$ & $6^{0}$ & $5^{0}$ & & & \\
\hline
\end{tabular}

La matriz final pone de relieve el papel preponderante que han alcanzado los entes dedicados al control de calidad en las funciones tradicionales (control de materiales, ejecución, proyecto y pruebas de servicio) las cuales obtienen las valoraciones superiores, si bien las otras tres posibles (asistencia técnica, control de entrega y organizativo) poseen pesos muy relevantes, con saltos sólo de 1 o 2 puntos porcentuales entre todas las funciones propuestas.

La cuantificación de los diferentes pesos entre las actividades o funciones del control propuestas por el modelo M153 es de utilidad para realizar comparativos con las diferentes planificaciones del control en la construcción de edificios de nueva planta.

Se desarrolla para ello una sencilla aplicación a través de la relación lineal entre el sumatorio de valoraciones del modelo para cada una de las funciones y el equivalente en la actuación a comprobar, la cual ha de evaluarse previamente.

De tal forma, si tomamos una de las promociones destacadas en el estudio y se evalúa por parte del equipo de trabajo las distintas funciones del control para este caso (en una escala proporcional al valor del modelo), obtenemos el cuadro comparativo que aparece en la Tabla 5.

El cociente entre las sumas de pesos sirve de coeficiente global que nos ilustra sobre la cercanía del proceso de control en el caso analizado con respecto al modelo M153. En el ejemplo expuesto el valor 0,81 indica gran similitud, lo cual es razonable si tenemos presente que se trata de uno de los expedientes del estudio con mayor alcance.

\section{CONCLUSIONES}

El estudio plantea una nueva organización de las actuaciones de control que desarrollan los diferentes entes de control de calidad en la construcción de edificios de nueva planta en España. Con ello los responsables del diseño y desarrollo del control (promotores y direcciones facultativas principalmente) pueden contar con una herramienta eficaz para cumplir con los objetivos propuestos y alcanzar la calidad.

La eficiencia del modelo viene avalada por un apoyo en la realidad contemporánea del sector, de donde se obtienen los factores condicionantes del control que las voces más cualificadas han marcado como hitos necesarios tras la experiencia acumulada. También de forma empírica (a través del análisis de 153 expedientes reales de control y la consulta a 17 expertos multidisciplinares) se ha derivado un conjunto de funciones operativas del control que debe ser suficiente para afrontar los citados factores. Se tiene así garantía, por otro lado, que se trata de intervenciones que pueden ser cubiertas por la mayor parte de las compañías de control de nuestro entorno.

El procedimiento metodológico multicriterio seguido ha utilizado una serie de técnicas conducentes a una valoración concreta de los parámetros de entrada mediante el método Delphi cuyo tratamiento y organización matricial con la herramienta Fuzzy-QFD condensa referencias numéricas a través de un reparto de pesos entre las funciones seleccionadas, ofreciendo también la distribución de estos pesos para cada uno de los factores.

Tabla 5. Cuadro de análisis comparativo con el modelo M153 para una promoción estudiada.

\begin{tabular}{|c|c|c|c|c|c|c|c|c|}
\hline & \multicolumn{7}{|c|}{ Funciones } & \multirow[b]{2}{*}{ Sumatorio } \\
\hline & $\begin{array}{l}\text { Control del } \\
\text { encargo }\end{array}$ & $\begin{array}{c}\text { Control del } \\
\text { proyecto }\end{array}$ & $\begin{array}{l}\text { Control de } \\
\text { materiales }\end{array}$ & $\begin{array}{l}\text { Control de } \\
\text { ejecución }\end{array}$ & $\begin{array}{l}\text { Pruebas de } \\
\text { servicio }\end{array}$ & $\begin{array}{c}\text { C. de } \\
\text { entrega y } \\
\text { posventa }\end{array}$ & $\begin{array}{l}\text { Asistencia } \\
\text { técnica }\end{array}$ & \\
\hline \multicolumn{9}{|l|}{ Modelo M153 } \\
\hline Evaluación Final & 295,0 & 423,1 & 480,1 & 497,4 & 467,5 & 349,7 & 371,8 & 2884,6 \\
\hline \multicolumn{9}{|l|}{ Caso estudiado } \\
\hline Puntuación equivalente & 167,2 & 394,9 & 400,1 & 439,4 & 397,4 & 227,3 & 322,2 & 2348,4 \\
\hline
\end{tabular}


El modelo así obtenido, denominado M153, nos dibuja un mapa de participación de las organizaciones de control con gran repercusión de las actuaciones clásicas (control de materiales, ejecución y proyecto). Si bien el resto de funciones poseen también un papel relevante, sin grandes diferencias de peso con las anteriores, lo que nos lleva a interpretar que el total de funciones elegidas deberían estar necesariamente presentes en el control de edificaciones de nueva planta.

En cuanto a los factores es destacable el papel trascendental que el modelo otorga a la independencia del controlador, situándolo a la cabeza en el orden de valoración, conjuntamente con los elementos más habituales en el desarrollo del control (muestreo, criterios de aceptación/rechazo, retroalimentación y coordinación entre etapas). Los dos factores restantes, vulnerabilidad y motivación, poseen cifras de evaluación no muy distantes a los anteriores, denotándose, por tanto, que todos los factores propuestos inciden de forma notable en la eficiencia del control.

A tenor de lo anterior, la forma en que se puede materializar un espectro de control con las características que el modelo M153 propone es materia para una investigación posterior, que queda abierta. En cualquier caso todo parece apuntar hacia un sistema de auditoría o certificación de edificio com- pleto, donde los agentes participantes desempeñen su contribución a la producción con toda la tipología de controles necesarios, mientras la figura externa de auditoría debe desarrollar una monitorización de todas las fases, ejecutando un control de contraste a modo de sanción definitiva (y no exento de su parte alícuota en la responsabilidad legal del proceso).

En la actualidad esta tipología de certificación, según el estudio realizado por Ramírez y Serpell (20), es muy escasa en nuestro entorno y no abarca la totalidad de aspectos relacionados con la calidad de la edificación ni posee tampoco una regulación legal expresa. En esta línea cabe citar también a las certificaciones de eficiencia energética, las cuales se han abierto camino en el sector inmobiliario durante los últimos años, con algunos ejemplos de notable prestigio que proyectan clara satisfacción a los usuarios (21), marcando quizás la pauta aplicable al resto de prestaciones y unidades constructivas del edificio.

\section{AGRADECIMIENTOS}

Los autores quieren dejar constancia de su agradecimiento al Dr. D. Antonio Garrido Hernández por el interés mostrado en este trabajo.

\section{REFERENCIAS}

(1) Garrido, A. (1995). Soluciones históricas al problema de la Calidad. En Aseguramiento de la calidad en la construcción (pp. 17-38). Madrid: ICCE.

(2) Ishikawa, K. (1994). Introducción al control de calidad, p. 61. Madrid: Díaz de Santos.

(3) Caballol, D. (2005). Metodología en la evaluación de riesgos en la construcción. Informes de la Construcción, 57(497): 47-52, doi: http://dx.doi.org/10.3989/ic.2005.v57.i497.467.

(4) Serra, J. (2005). La normativa y la reglamentación en la mejora de la calidad en la construcción y su relación con la innovación. Informes de la Construcción, 57(499-500): 78-86, doi: http://dx.doi.org/10.3989/ic.2005.v57.i499-500.484.

(5) Osorio, J. C., Arango, D. C., Ruales, C. E. (2011). Selección de proveedores usando el despliegue de la función de calidad difusa. Revista EIA, Escuela de Ingeniería de Antioquia, 8(15): 73-83.

(6) Zadeh, L. (1965). Fuzzy Sets. Information and Control, 8(3): 338-353, doi: http://dx.doi.org/10.1016/Soo199958(65)90241-X.

(7) García-Meseguer, A (2001). Fundamentos de calidad en construcción. Sevilla: Fundación Cultural del Colegio Oficial de Aparejadores y Arquitectos Técnicos de Sevilla.

(8) Macías-Bernal, J. M., Calama-Rodríguez, J. M., Chávez-de Diego, M. J. (2014). Modelo de predicción de la vida útil de la edificación patrimonial a partir de la lógica difusa. Informes de la Construcción, 66(533): eoo6, doi: http://dx.doi. org/10.3989/ic.12.107.

(9) Monjo-Carrió, J. (2007) Durabilidad vs Vulnerabilidad. Informes de la Construcción, 59(507): 43-58, doi: http://dx.doi. org/ 10.3989/ic.2007.v59.i507.531.

(10) Garrido, A. (2004). El Libro del Director de la Ejecución de la Obra, p. 136. Madrid: Leynfor Siglo XXI.

(11) González-Pachón, V., Moncada, J. C. (2007). El plan de Control de Calidad y el Código Técnico. Arte y Cemento, 2007(2067): 10-20.

(12) Navarro, E. (2008). Revisión de la Motivación de los Trabajadores de la Construcción: 1968-2008. Revista de la Construcción, 7(02): 17-29. http://www.redalyc.org/articulo.oa?id=127612584002. ISSN: 0717-7925.

(13) García-Meseguer, A. (1972). Primer Congreso Nacional de la Calidad (febrero de 1972). Panorama español actual del control de calidad en la construcción. Informes de la construcción, 25(241): 79-85, doi: http://dx.doi.org/10.3989/ ic.1972.v25.i241.3228.

(14) Calavera, J. (1984). Introducción a las técnicas de Control de Calidad. En Arenas, J. J. (Ed.), La calidad en la edificación. Santander: Universidad de Santander.

(15) Viéitez, J. A., Ramírez, J. L. (1984). Patología de la construcción en España: Aproximación estadística. Informes de la Construcción, 36(364): 5-15, doi: http://dx.doi.org/10.3989/ic.1984.v36.i364.1901.

(16) Conradi, E. (2002). Evaluación de la Calidad actual de los Proyectos de Edificación. Propuesta de control mediante implantación de Sistemas de Gestión de Calidad (Tesis doctoral). Sevilla: Universidad de Sevilla.

(17) Bevilacqua, M., Ciarapica, F. E., Giacchetta, G. (2006). A fuzzy-QFD approach to supplier selection. Journal of Purchasing \& Supply Management, 12(1): 14-27, doi: http://dx.doi.org/10.1016/j.pursup.2006.02.001. 
(18) Singhaputtangkul, N., Low, S. P., Teo, A. L., Hwang, B.-G. (2013). Knowledge-based Decision Support System Quality Function Deployment (KBDSS-QFD) tool for assessment of building envelopes. Automation in Construction, 35(2013): 314-328, doi: http://dx.doi.org/10.1016/j.autcon.2013.05.017.

(19) Instituto de Microelectrónica de Sevilla (IMSE-CNM) (2012). Herramientas de cad para lógica difusa: http://www2. imse-cnm.csic.es/Xfuzzy/Xfuzzy_3.o/download_sp.html. Sevilla.

(20) Ramírez, V., Serpell, A. (2012). Certificación de la calidad de viviendas en Chile: Análisis comparativo con sistemas internacionales. Revista de la Construcción, 11(1): 134-144, doi: http://dx.doi.org/10.4067/So718-915X2012000100012.

(21) Goins, J., Moezzi, M. (2013). Linking occupant complaints to building performance. Revista Building Research \& Information, 41(3), 361-372, doi: http://dx.doi.org/10.1080/09613218.2013.763714. 